AGH DRILLING, OIL, GAS • Vol. $29 \cdot$ No. $3 \cdot 2012$

http://dx.doi.org/10.7494/drill.2012.29.3.419

\author{
Maciej Lorenc*, Witold Warowny**
}

\title{
APPARATUS TO STUDY VOLUMETRIC PROPERTIES OF THE GAS HYDRATES
}

\section{INTRODUCTION}

Previous studies describe gas hydrate in sufficient detail the process in many ways: the properties, changes in the physical structure of the crystal lattice or usability and prevent the phenomenon of hydration. In the literature, many studies have been published and presented numerous experimental models of computing, in particular the share of hydrocarbons and carbon dioxide [1, 2]. These data provide information on the pressure and temperature of nucleation, the growth rate of hydrates, the morphology of the crystal lattice, the conditions for the filling gas, and the influence of other factors on the process. Several studies also relates to the use of the properties of the hydrates in practical applications. Made a lot of research on the phenomenon of hydration in natural gas and its components, including extensively studied experimentally possible preventive hydration for the different stages of the technology in the oil and gas industry, and the positive use of the properties of hydrates. An example of the application of the phenomenon of hydration can be well understood and described in the literature transportation and storage of natural gas, which are summarized in the article Warowny and Lorenc [3]. Still in the natural gas industry, some issues are still open, or require complete experimental knowledge. So far, insufficient knowledge in the context of the separation of gaseous mixtures of acidic components, especially hydrogen sulfide. [4] This component is relatively common in the natural gas, and its highly toxic nature, make it necessary to eliminate it from the gas stream, to trace amounts of normative [5].

* Polish Oil and Gas Company in Warsaw (PGNiG), Branch of Geology and Exploitation, Reservoir Engineering Department in Sanok, Poland

** Warsaw University of Technology, Department of Civil Engineering, Mechanics and Petrochemistry, Poland 
Strong hydrogen sulfide hydrate-forming properties as compared to other natural gas components make it possible to be removed from the gas mixture, which directs the subject [6] and experimental research in the AGH University of Science and Technology. For this purpose, a preliminary study was carried out, the results suggest the potential use of this method in the gas industry [7,8]. As a result of the research project on gas desulfurization method of gas hydrate, sponsored by the Polish Oil and Gas Company (PGNiG S.A.) was created at the Academy of Science and Technology laboratory equipped with pVT apparatus designed in order to study the properties of gas hydrates. Some technical solutions applied in this apparatus are described in the literature, but are also innovative solutions such as the reactor volume change mechanism or system for automatic sampling with lossless nafion-drying method.

This paper presents a comparison of selective measurement techniques used in research laboratories with details of their potential advantages and disadvantages with the current at AGH University of Science and Technology in Cracow measuring apparatus.

\section{ELEMENTS OF THE APPARATUS}

Generally pVT apparatus to study the phenomenon of hydration in most cases is composed of the following elements: 1) the tank ,reactionary”, 2) sample preparation system, 3) auxiliary systems (vacuum, pressure and thermostatic), 4) filling system (sampling system to research), 5) monitoring and control system, 6) physicochemical properties measuring apparatus included in the general and 7) data collection remote system. Almost always there are additional elements to extend the functionality of the equipment or duplicate measurements in order to verify and increase the precision of the results.

A representative example of above set of elements of the apparatus can be a version which built in the Department of Drilling, Oil and Gas, AGH UST in Cracow, with the intention of study sulfur natural gas hydration. Schematic diagram of the apparatus is shown in Figure 1, which contains the basic elements listed above. The apparatus AGH UST has several new elements to extend its functionality: 1) shown in Figure 2, a mechanical assembly for changing the volume, 2) the additional piston increasing pressure of the sample, 3) a separator system, and an auxiliary tank for the returning sour water to process, 4) an automatic collection and sample drying lossless system with automatic chromatographic measurement, 5) shown in Figure 4 cylinder reactor design allows to maintain a constant temperature inside. Each experimental apparatus design is directed in terms of their construction and size for the intended purpose of the research. For example, the crystal structure morphology of hydrates investigations do not require the large size of the reaction vessel, in which the elements of the observation and/or detection techniques applied to an essential part of the apparatus. 
Basic detection techniques are the methods: NMR (Proton Nuclear Magnetic Resonances) $[9,10]$, XRD (X-Ray Diffraction), Raman spectroscopy [10, 11], neutron scattering [12] and microscopic examination.

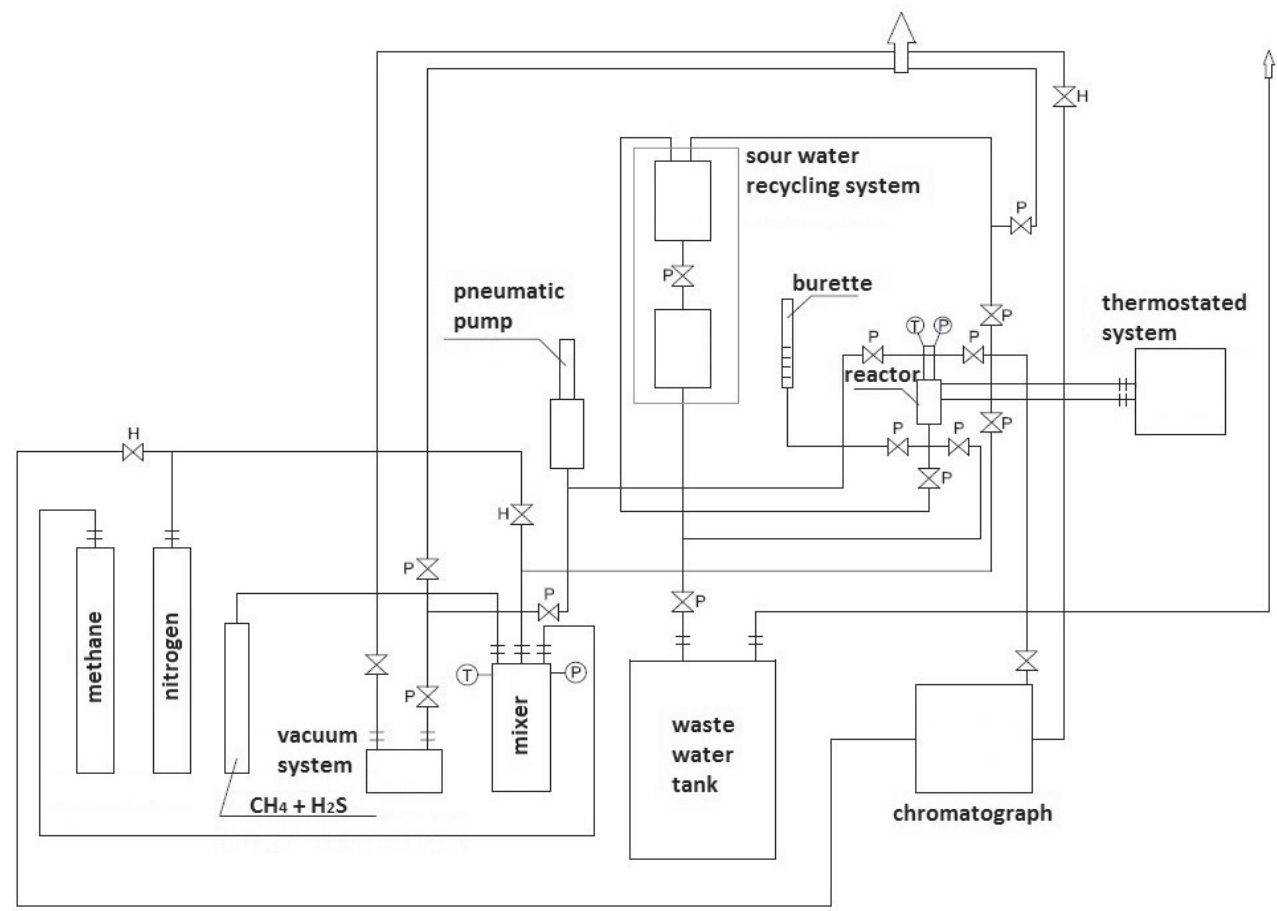

Fig. 1. Schematic diagram of the apparatus to study the hydration of sulfurized natural gas in the AGH UST in Cracow

Studies to determine the conditions and limitations the production process of methane hydrate to its subsequent transport and storage of required equipment as close to the construction and size of the production system (industrial) [13], which designs are optimal in terms of rate and amount of production of hydrates. In such a process for its control is generally used parameters such as pressure, temperature and stirring speed and the residence time in the reactor. Comparison of laboratory to semi-industrial plant is usually burdened significant error, because it is difficult to take into account change the scale factor of the process, which introduces the effects can not be observed in the small-sized reactors, such as the product flows in the system, optimization of the process in relation to energy requirements, control the amount of substrates supplied to the reactor, maintaining the continuity of the process, and more. Thus, there is a need and a tendency of building large-bulky research installations, in order to eliminate the aforementioned problems. Most of this type of laboratory apparatus has in addition to orientation the peculiar element distinguishing them, which leads to a new quality of results. A number constructions have been described by Lorenc and Warowny in the conference paper [14]. 


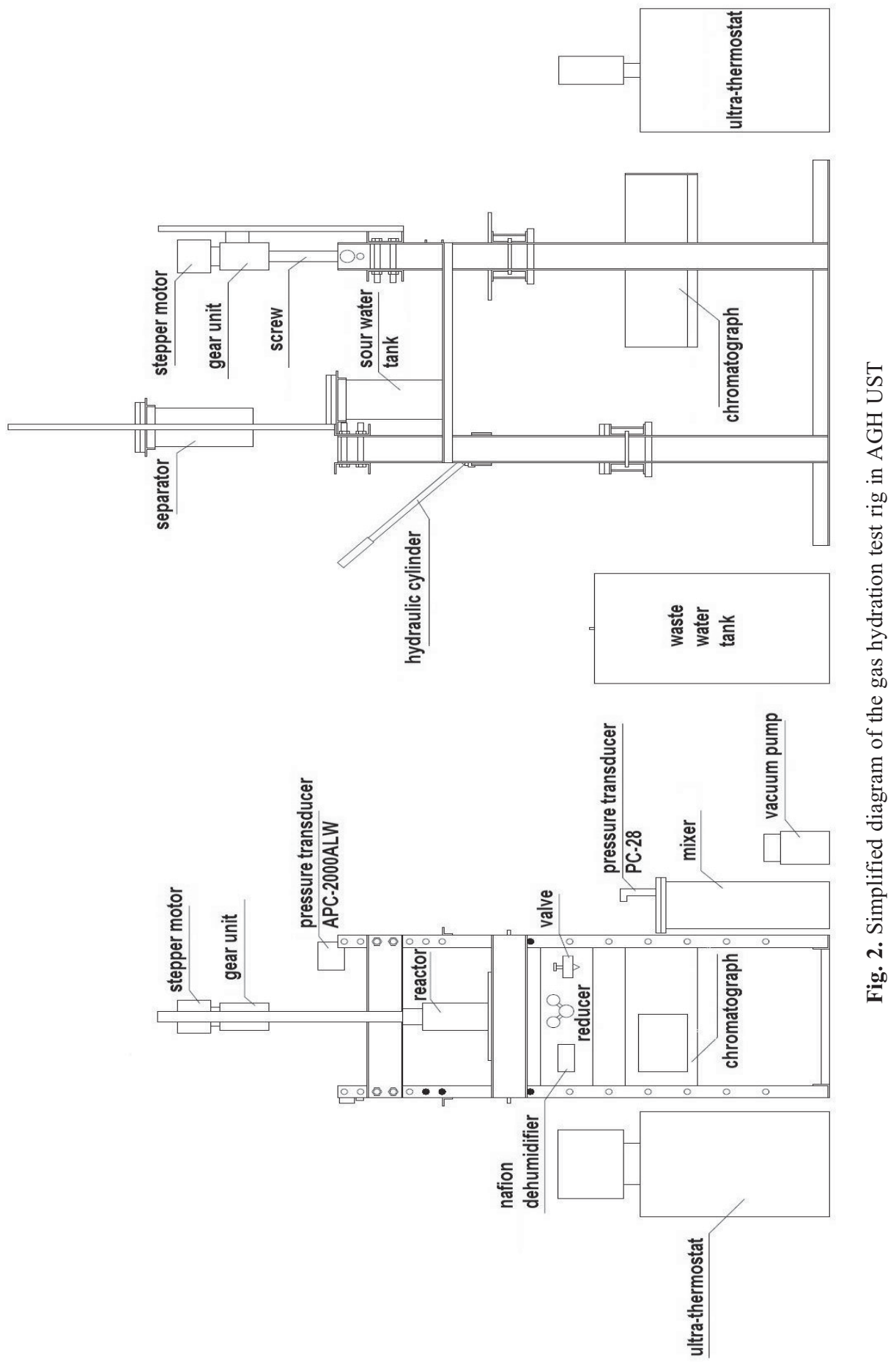




\section{TYPES OF REACTORS}

Hydration reactors, which are the main construction element of the apparatus, because of the research method are made of different materials, have different designs and sizes. Many of the design characteristics of the hydration tanks and methodology include: normal pressure vessels made of stainless steel, through tanks with mixing mechanism, tanks with quartz or sapphire windows (allowing the observation of the process), vessels made of plastic or quartz glass, piston type design of vessels (floating or fixed piston) and many other outstanding varieties more or less modification of the above. One important difference is the method of filling the reactor substrates.

The design "reactor" of hydration located in AGH UST was built on the basis of its own technical design. The reactor has a variable volume chamber, carried by a piston coupled to the stepper motor. The mechanical assembly of feed rate plunger as shown in Figure 3, which is a fully automated system as a function reducing the volume of the gas pressure decreasing. The reactor was equipped with four quartz windows allowing the laser measurement of the turbidity of fluid in the two planes. Figure 4 shows a view of the aforementioned construction of a reactor built in the University. Mixing method was used in the present apparatus is commonly used, and uses a magnetic coupling to the transmission element within the mixing chamber.

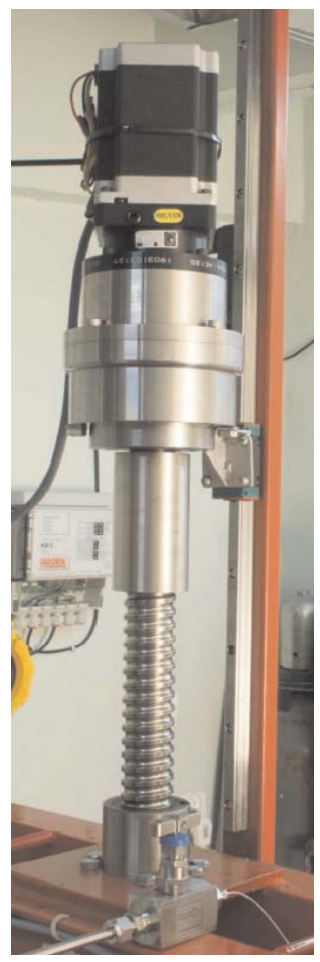

Fig. 3. The mechanical assembly of feed rate plunger 


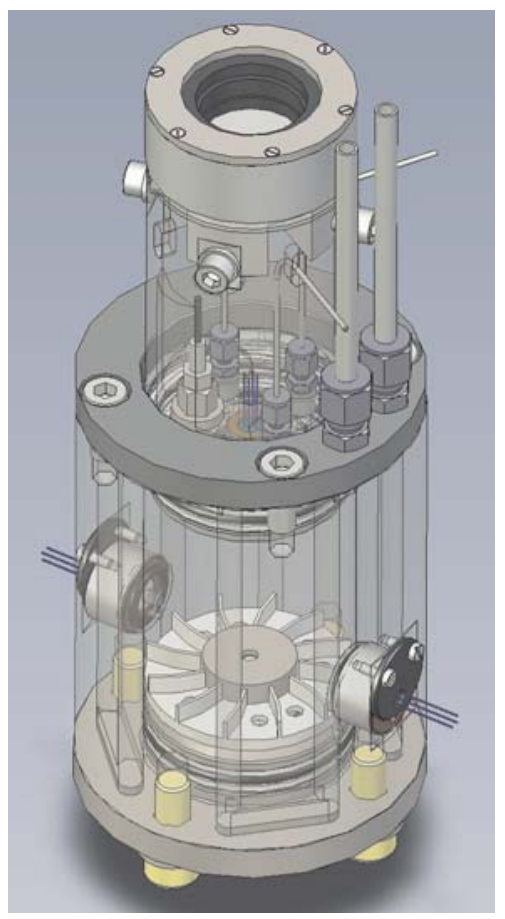

Fig. 4. "Reactor" of hydration built in the AGH UST in Cracow

\section{PREPARATION OF THE SAMPLE}

Depending on the type of reactor, preparing a water-gas mixture can take place in two ways: the gas filling of the reactor previously filled with the appropriate amount of water (most cases) or the injection of water to a previously filled reactor with gas as shown in Figure 5 [15].

Apparatus located on the AGH UST is equipped with the first of the above methods of filling the reactor substrates due to their small size and the impossibility application of the hydraulic system.

Preparation of the test sample in the gas phase can be done in two ways: the preparation of the gas mixture in a separate container, which is related to the time required for stabilization, or by direct application to the reactor chamber gas substrates (unstable mixture). The advantage of the first method is a stable mixture of known composition, and the disadvantage is the time required for stabilization, which often considerably extends the process of preparing the sample as opposed to the other methods. The disadvantage of the second method is not known until the end of the mixture composition, which locally may be slightly different from the expected global composition. In the case of apparatus AGH UST problem is eliminated because the apparatus is equipped with a mixer, to which 
the gas substrates are fill before. After a predetermined stabilization time after mixing, before the gas is provided to the reactor, the mixture is examined by chromatography on a device's Chromatotec H2SMEDOR. Chromatographic measurement will also be conducted during the measurement automatically, as described in section 6. Integrated with the computer unit is dedicated to the chromatographic determination of hydrogen sulfide in methane in the smallest of the measurement range of $20 \mathrm{ppb}$, whereas the largest, up to $4 \%$. The time to obtain a result its only 60 seconds. A small volume of sample is collected automatically by the chromatograph does not make a large loss of pressure in the reactor chamber. The reported results are time stamped. The software provided by the manufacturer chromatograph allows to calculate the retention time, peak area and other parameters automatically. The device has a built-in monitor which enables the visualization of results and record data on a separate PC or data logger. Chromatograph software is also prepared for the conversion, calibration, or export the data and configuration settings for the measurement.

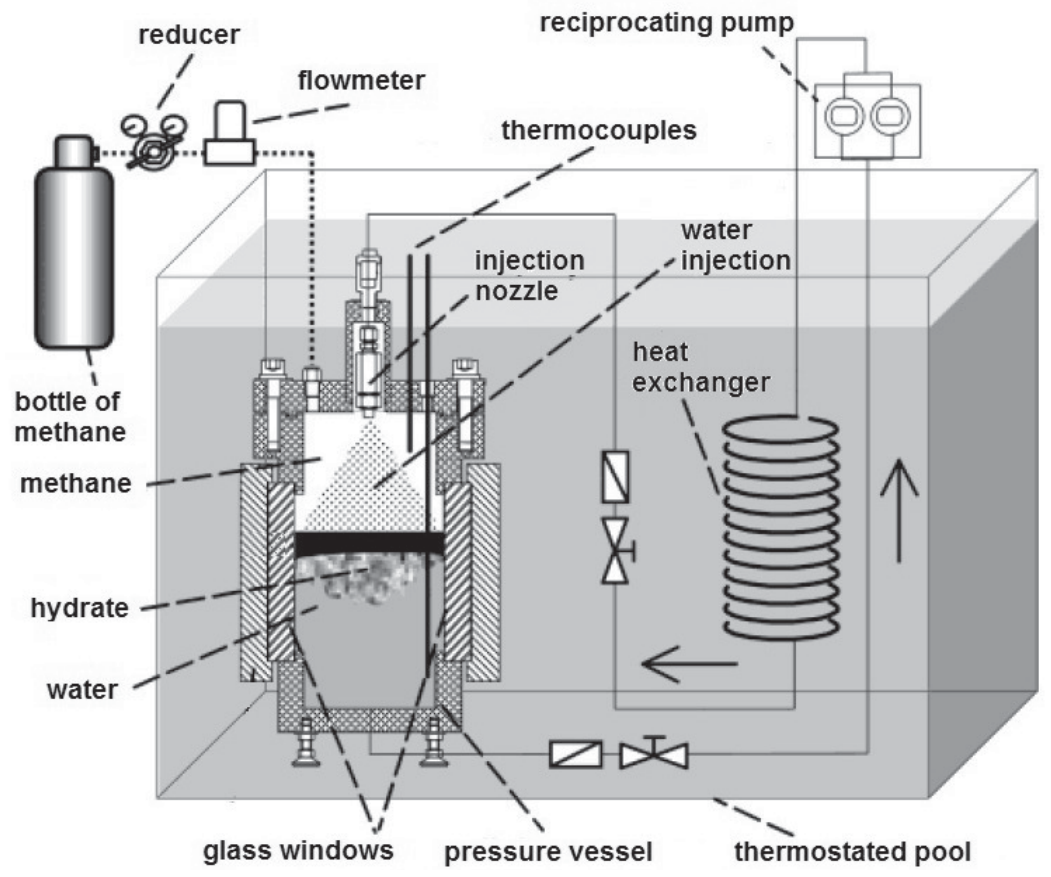

Fig. 5. The injection method of water supply to the reactor

\section{NUCLEATION POINT MEASUREMENT}

The moment of the beginning of nucleation occurs in a volume of liquid through the formation of a single unstable crystal hydrate, without change of thermodynamic parameters of the mixture such as pressure $p$ and temperature $T$. In the literature, often can be found 
a description of the initiation of nucleation process by measuring the pressure drop and slight temperature increase in hydrate environment (hydrate formation process is an exothermic process). This interpretation of phenomenon is incorrect, because the changes in both parameters $p$ and $T$ indicate the agglomeration of hydrate crystals previously created in the liquid. Therefore, the measurement of nucleation point, namely designation phase equilibrium hydrate-gas-water requires the use of more sophisticated methods than measurement of the parameters $p$ and $T$. For this purpose are the previously mentioned techniques: NMR (Proton Nuclear Magnetic Resonance), XRD (X-Ray Diffraction), Raman spectroscopy, neutron scattering on the crystal lattice or microscopic observation and the simplest of them, the measurement of the turbidity of the fluid.

The last above methods of nucleation point measurement used in the apparatus was built on the AGH UST. The method has been implemented with the use of electronic modules that contain semiconductor laser and detector. The reactor has a mirrored turbidity measurement in two dimensions as shown in Figure 4. There are diametrically opposed lying a compact design modules (transmitter-receiver) in both surfaces allowing the simultaneous measurement of two parallel laser beams. Turbidity measurement is the detection of a weak laser light, in relation to the homogeneous phase, when disorders, type of nucleation newly created particles, appears. This phenomenon occurs when measuring the intensity of light passing through the liquid just below the separation surface of the gas phase. In addition to mutually verify measurements from the underlying parallel laser beams were also performed two laser-detector modules lying perpendicular to the surface of the interface water-gas system, giving a total four mutually verify the fluid turbidity measurements.

\section{SAMPLING SYSTEM}

Much of the research on the conditions for gas hydrate formation is based on the production in the reactor vessel and the pressure and temperature measurement of hydration. Such studies does not require the sampling of gas remaining from the creation of a hydrate, because it is not usually subject verification mixture composition. Is often used to chromatography measure the composition of the gas sample before and after hydration, indicating initial and final compositions of the gas mixture. Another group of studies are using current measure of chemical composition of the sample during hydration. Very good results were obtained from these measurements using the NMR technique to study the structure of the formation of hydrates SII of methane and propane mixture [9]. NMR measurement was included in both the hydrate and gas surrounding. This method showed a varied loss of components of the mixture with respect to the associated increase in the amounts of these components in the hydrate phase. The advantage of this method is that no sampling during the hydration process, and the disadvantage is the small volume of the sample and the absence of stirring, and a number of limitations associated with the use of NMR techniques. Similar results to those described above can be achieved using an automated sampling sys- 
tem from the reactor volume to chromatography, a technique is fraught with greater measurement error, which is mainly reduced due to larger ratio of sample volume compared to the usage volume of reactor. The pressure drop due to the loss of gas from the reactor chamber can also be compensated by reducing the usage volume the appropriate value calculated from the measured parameters $p$ and $T$.

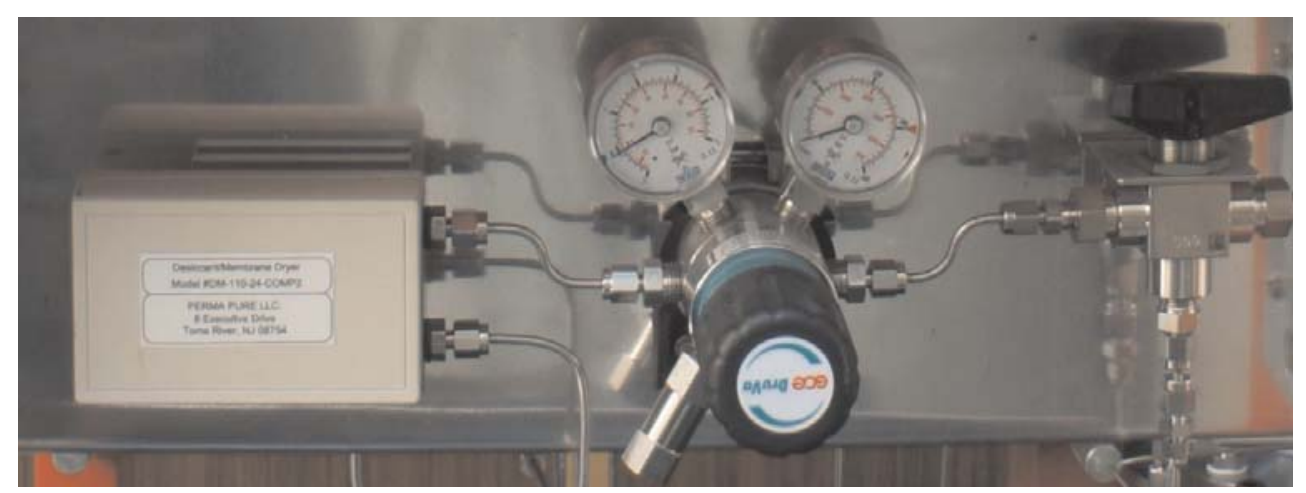

Fig. 6. Automatic sampling system

The above mentioned sample system used in the apparatus built in the AGH UST. Shown in Figure 6, set of devices includes sequentially from right: valve, pressure reducer and nafion dryer. The automatic valve is included building chromatograph. Control the amount of sample gas consumption is a computer chromatograph respectively open and close the automatic valve. Collected amount of gas results in lower pressure in the reactor chamber, which is automatically converted to feed rate of the piston and an appropriate calculated value of the usage volume is reduced.

Humid gas sample must be dried to a value strictly specified by the manufacturer, before it goes into the gas chromatograph. The problem of drying in the laboratory, because of small volume of gas collected is associated with secondary difficulties, the possibility of loss of gas from the sample. The problem is of particular importance not only when the sample volume is small and the even greater if the gas components are well soluble in water. An innovative device used in the apparatus AGH UST is the previously mentioned nafion drying gas sample. The relatively large difference between the solubility of methane and hydrogen sulphide in combination with the small volume of the sample gas results in a large measurement error, due to the solubility of the sample components. This error can be eliminated almost entirely using nafion drying marked schematically in Figure 7. The dryer consists of a nafion tubes through which gas flows, gas washes receiving moisture from their surfaces. The principle of operation is based on Nafion absorption in the material tube, the active substance is a sulfonic acid. The advantage is that the rate of drying, because to achieve the desired dew point just one second, which allows for a short residence time of gas in 
the tubes and thus drastically reduce (decrease) the size of the drying device. The selectivity of drying both to methane and for hydrogen sulfide is complete. This means that during the absorption of water molecules mentioned above, none of the gas molecules can not be accidentally absorbed. In addition, the sample gas can be heated up to temperatures far in excess of $100^{\circ} \mathrm{C}$, which is not negligible in the case of samples with hydrogen sulfide, because it prevents the of hydrate plugs. The disadvantage of nafion dehumidifiers is low pressure limited by value 0.54 bar, which is the value applies to the mechanical stability of the nafion tubes.

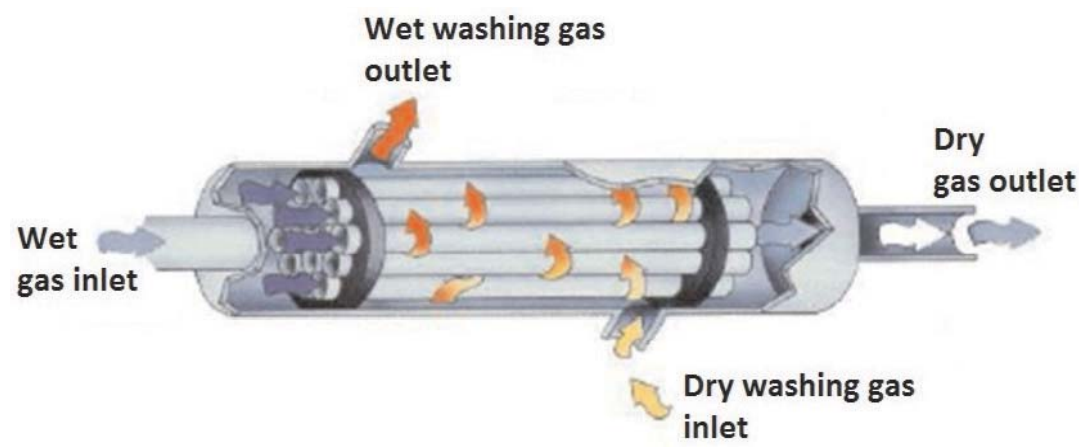

Fig. 7. Schematic of nafion dehumidify

\section{STABILITY OF THERMODYNAMIC CONDITIONS}

Experimental studies using pVT method in all cases related to the measurement parameters of state of the sample, as well as the control/regulation of these parameters. Successful experimental work depends largely on the accuracy of measurement and precision of control the pressure and temperature of the sample. In the literature, several solutions to the methodology specified in this field, from pools with thermostatting fluid through coils wrapped around the reactor to the special design of the reactor jackets. Most of the temperature control system has a similar operating parameters for precision temperature maintained. The difference appears primarily in the speed and achieve the desired temperature and thermal inertia, i.e., the temperature change rate, that is the larger the volume of liquid thermostatic system the greater the inertia and higher power required to change the temperature. The advantage of this condition is better stabilization system conditions, while the disadvantage of slower temperature changes.

The AGH UST apparatus was equipped with ultra-thermostat UTU-6 shown in Figure 8 , both for heating a thermostatic medium and of its cooling to a temperature of $-12^{\circ} \mathrm{C}$.

Thermostatic medium inside the ultra-thermostat UTU-6 is a glycol, which is a set temperature with an accuracy of $0.5^{\circ} \mathrm{C}$ is pumped through a set rate to circulation channels in the reactor body. This solution enables fast reactor body temperature control, and therefore inside the sample. 


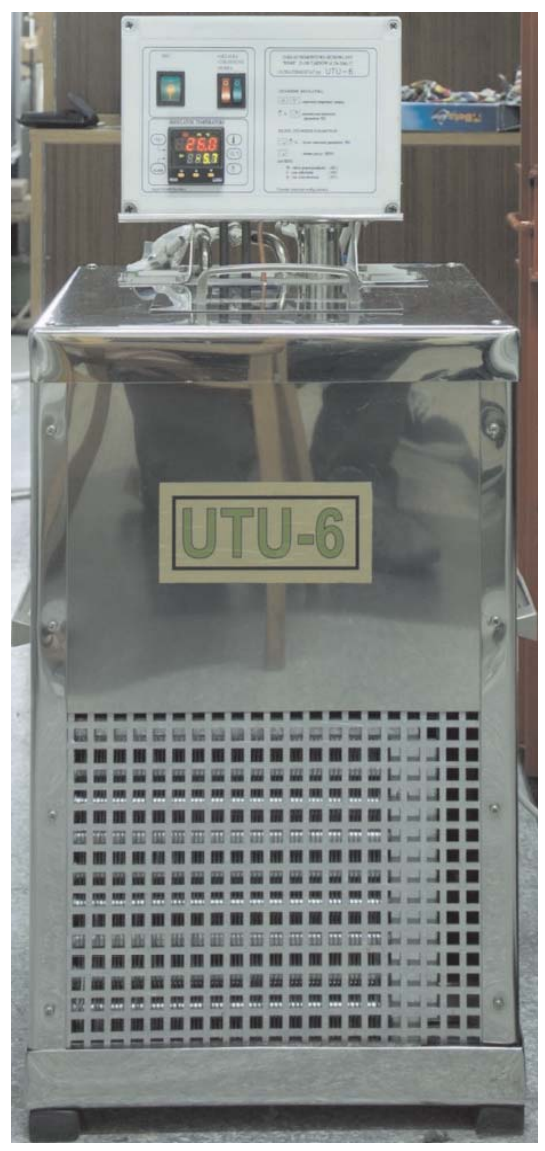

Fig. 8. Ultra-thermostat UTU-6

\section{SUMMARY}

Wide range of gas hydrate theme necessitated a lot of research laboratories with less or more complicated measurement equipment. Due to the nature of experimental research, building apparatus are directed to achieve the planned goals (results). Yet there is no universal pVT apparatus for testing gas hydrate, even in the aspect of quality. The reason for this is the fact that some types of experiments can not be carried out in parallel, because they require an individual approach. Determination of phase equilibrium (hydrate dissociation point) and after nucleation with use mentioned in this article, the advanced measurement techniques is closely linked with additional studies such as the morphology of crystals. Such research will almost always do not allow parallel measurement the selective separation of the substance or reaction kinetics during mixing. This problem can be reduced 
partly by modifying the reactor through appropriate measuring devices and do a program of corresponding sequence of measurements. This approach to the problem of multi-functionality test equipment designing and constructing taken in the laboratory at AGH UST. Determination of phase equilibrium and nucleation point precedes the second stage of research related to the measurement of the selective separation of gas mixture components.

Research project for the selective removal of hydrogen sulfide from a mixture with methane, using the phenomenon of hydration resulted in the building pVT apparatus directed for testing many different properties of the gas hydration process which are in the range of operating parameters of the apparatus. An extensive experimental program for the project is open to further study the phenomenon of hydration cycle in the laboratory AGH UST in Cracow.

\section{REFERENCES}

[1] Ding T.: Gas Hydrates to Capture and Sequester $\mathrm{CO}_{2}$. Dave C. Swalm School of Chemical Engineering, Mississippi 2004 (M.Sc. Thesis).

[2] Seo Y-T., Lee H.: Multiple-Phase Hydrate Equilibria of the Ternary Carbon Dioxide, Methane, and Water Mixtures. The Journal of Physical Chemistry B, 105, 2001, pp. 10084-10090.

[3] Warowny W., Lorenc M.: Hydraty w transporcie i magazynowaniu gazu ziemnego. Gaz, Woda i Technika Sanitarna, 80 (10), 2006, pp. 24-31.

[4] Lorenc M.: Separacja kwaśnych składników mieszanin gazowych z wykorzystaniem zjawiska hydratacji. Gaz, Woda i Technika Sanitarna, 81 (1), 2007, pp. 2-9.

[5] Lorenc M.: Hydraty w transporcie gazu. Rurociagi, 1 (47), 2007, pp. 3-11.

[6] Lorenc M., Warowny W.: Odsiarczanie gazu ziemnego metoda hydratacji. Nafta-Gaz, 70 (2), 2009, pp. 1-10.

[7] Kamata Y. i inni: Hydrogen sulfide separation using tetra-n-butyl ammonium bromide semi-clathrate (TBAB) hydrate. Energy \& Fuels, 19, 2005, pp. 1717-1722.

[8] Shiojiri K.: A new proces for the separation of condensable greenhouse gases by the formation of clathrate hydrates. Vision, The bimonthly e-journal of the World Student Community for Sustainable Development (www.wscsd.org/ejournal/), 2004.

[9] Kini R.A., Dec S.F., Sloan E.D. Jr.: Methane + Propane Structure II Hydrate Formation Kinetics. The Journal of Physical Chemistry A, 108, 2004, pp. 9550-9556.

[10] Sloan E.D. Jr.: COMMENTARIES, Clathrate Hydrates: The Other Common Solid Water Phase. Industrial and Engineering Chemistry Research, 39, 2000, pp. 31233129.

[11] Uchida T., Okabe R., Mae S., Ebinuma T., Narita H.: In Situ Observations of Methane Hydrate Formation Mechanisms by Raman Spectroscopy. Annals of the New York Academy of Sciences (www.blackwell-synergy.com - Gas Hydrates: Challenges For The Future), 912, 2000, pp. 593-601. 
[12] Hutter J.L., King H.E. Jr., Lin M.Y.: Polymeric Hydrate-Inhibitor Adsorption Measured by Neutron Scattering. Macromolecules, 33, 2000, pp. 2670-2679.

[13] Fitzgerald A.: SPE Europe Conference. Aberdeen, 4-7 September 2001.

[14] Lorenc M., Warowny W.: Doświadczalne metody badan hydratów gazowych. Wiertnictwo Nafta Gaz, 28 (1-2), 2011, pp. 227-251.

[15] Tsuji H., Ohmura R., Mori Y.H.: Forming Structure-H Hydrates Using Water Spraying in Methane Gas: Effects of Chemical Species of Large-Molecule Guest Substances. Energy \& Fuels, 18, 2004, pp. 418-424. 